\title{
Autonomic dysreflexia caused by cervical stenosis
}

\author{
Krishn Khanna $^{1} \cdot$ Alexander A. Theologis $^{1} \cdot$ Bobby Tay $^{1}$ \\ Received: 6 July 2017 / Revised: 4 September 2017 / Accepted: 7 September 2017 \\ (C) International Spinal Cord Society 2017
}

\begin{abstract}
Introduction Autonomic dysreflexia (AD) is a well-known sequela of high spinal cord injuries (SCI). The characteristic episodic presentation is one of increased sympathetic tone: diaphoresis, hypertension, tachycardia, or reflex bradycardia. The episodes are triggered by visceral sensations and can last days to weeks.
\end{abstract}

Case presentation This report presents the case of a 73-year-old male with cervical stenosis, with a longstanding history of "hot flashes" accompanied by dizziness, flushing and diaphoresis, and palpitations. The patient was evaluated extensively by cardiology, endocrinology, and neurology with no treatable pathology determined aside from the patient's cervical stenosis. The patient was diagnosed with autonomic dysreflexia caused by cervical spinal stenosis and underwent anterior cervical decompression and fusion (ACDF) at the stenotic C5-C6 level. He found near complete resolution of his autonomic symptoms.

Discussion We hypothesize that the cervical compression caused a disruption in the regulatory control of the sympathetic preganglionic neurons resulting in the autonomic symptoms. Although numerous studies exist of patients with a traumatic onset of $\mathrm{AD}$, to the best of our knowledge, this is the first case report in the literature of autonomic symptoms that stemmed from cervical stenosis. The purpose of this case report is to alert clinicians to a potential association between $\mathrm{AD}$ and spinal stenosis, which may exist outside the realm of SCI.

\section{Introduction}

Autonomic dysreflexia (AD) is a well-known sequela of high spinal cord injuries (SCI) [1-3], known to be present in over $70 \%$ of complete injuries [4]. The episodic nature of the syndrome consists of a constellation of autonomic and cardiovascular symptoms, which can be as benign as anxiety and headaches to life-threatening emergencies such as malignant hypertension and cardiac arrhythmias. AD is typically seen in SCIs at or cephalad to the T6 level. The characteristic presentation is one of increased sympathetic tone: diaphoresis, an elevation in blood pressure $20-30 \mathrm{~mm}$ $\mathrm{Hg}$ above the patient's systolic baseline, usually accompanied by bradycardia, though tachycardia and atrial fibrillation can also be present [1,5]. The severity of symptoms is dependent on both the spinal level [6] and

Krishn Khanna

krishn.khanna@ucsf.edu

1 Department of Orthopedic Surgery, University of California, San Francisco, CA 94143, USA severity of injury [7], with more cephalad and more complete injuries leading to greater deviation from homeostasis. The episodes are triggered by visceral sensations and may last days to weeks [1].

This report presents the case of a patient with single-level cervical stenosis, with a longstanding history of "hot flashes" accompanied by dizziness, flushing, diaphoresis, and palpitations. The patient was evaluated extensively by cardiology, endocrinology, and neurology with no treatable pathology determined aside from the patient's cervical stenosis. The patient was diagnosed with AD caused by cervical spinal stenosis and underwent anterior cervical decompression and fusion (ACDF) at the stenotic C5-C6 level to treat his radicular symptoms, which resulted in near complete resolution of his autonomic symptoms. Although numerous studies exist of patients with a traumatic onset of $\mathrm{AD}$, to the best of our knowledge, this is the first case report in the literature of AD-like symptoms that stemmed from cervical stenosis. The purpose of this case report is to alert clinicians to a potential association between $\mathrm{AD}$ and spinal stenosis. 


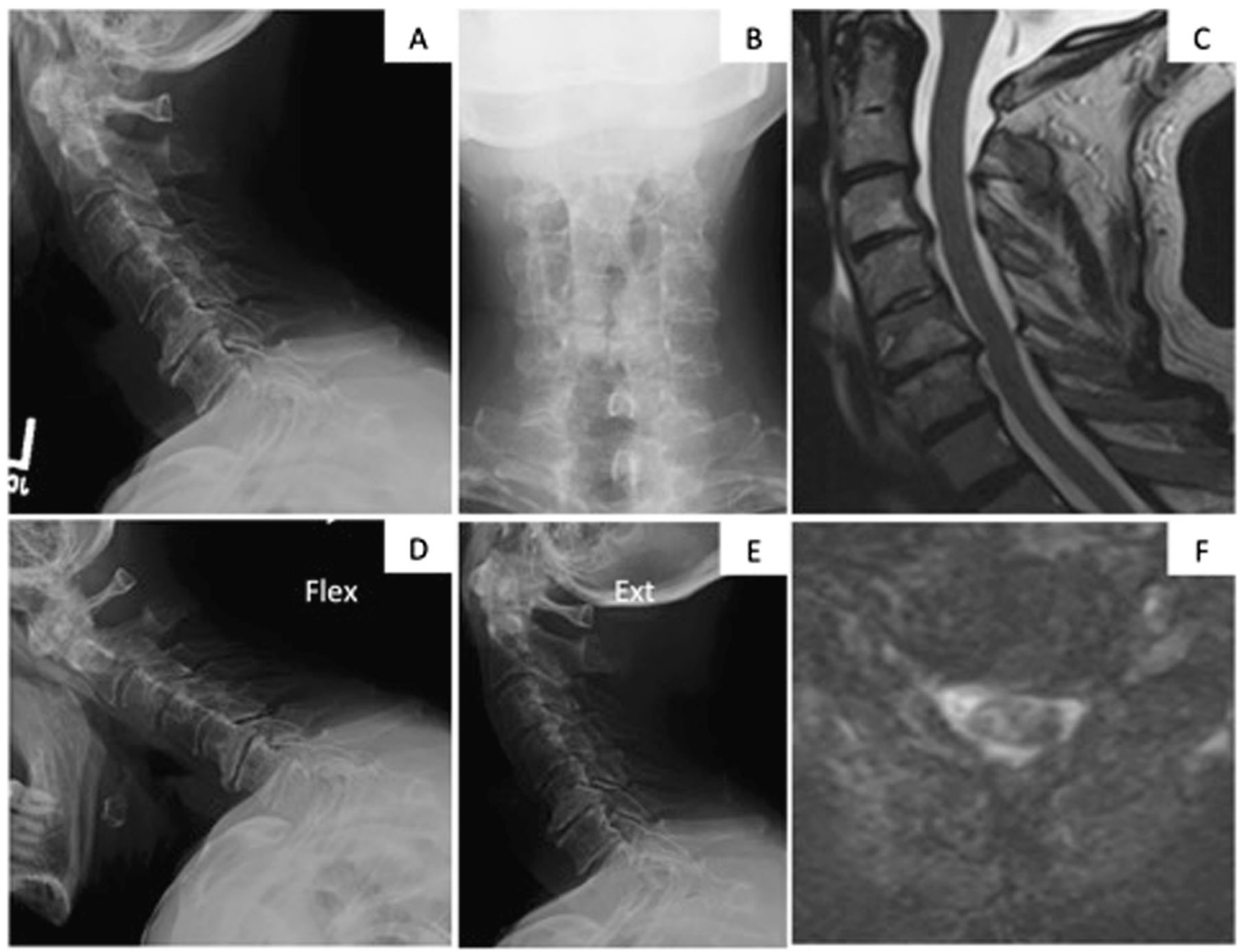

Fig. 1 Initial cervical spine radiographs $(\mathbf{a}, \mathbf{b}, \mathbf{d}, \mathbf{e})$, and MRI $(\mathbf{c}, \mathbf{f})$ in 2014 demonstrate multi-level degenerative disc disease with mild/moderate central and bilateral foraminal stenosis at $\mathrm{C} 5-6$

\section{Case presentation}

\section{Patient history}

The patient is a 73-year-old male with a history of hypertension, sleep apnea, prostate cancer, and prior L3-5 laminectomy with L4-5 posterior instrumented spinal fusion for spinal stenosis and degenerative spondylolisthesis 7 years prior. 4 years after the operation he re-presented to the clinic reporting right-sided neck pain that radiated into the right scapula. There was no radicular pain into the arm. Radiography and magnetic resonance imaging (MRI) at that time (2014) demonstrated degenerative disc disease at C5-6 with neuroforaminal narrowing bilaterally at C5-6 and C6-7 and right neuroforaminal narrowing at C3-4 (Fig. 1). He was sent for a selective nerve root injection on the right at $\mathrm{C} 4$, which provided lasting pain relief for several years.

\section{History of presenting illness}

As part of his urologic surveillance for prostate cancer 2 years ago, the patient first reported that he suffered "hot flashes" for several years. Over the next several months, he noted that these "hot flashes" became more intense and more frequent. In addition to night sweats and clammy hands, feet, and back, he reported severe fatigue, dizziness, lack of focus and mental clarity, his skin turning red, and tachycardia during these flashes. The episodes were cyclicsymptoms lasted up to 5-10 days with intervals of asymptomatic periods for several days. He was evaluated by his cardiologist and primary care doctor for these symptoms and diagnosed with paroxysmal atrial fibrillation. He was placed on anticoagulation with aspirin and atenolol for rate control. Over the next year, he continued to describe similar symptoms, including chills, intermittent tremors, blurry vision, clammy hands and feet, and wide fluctuations of blood pressure.

An extensive evaluation for the etiology of these symptoms was undertaken by an endocrinologist in 18 months after the initial report. Levels of prolactin, Follicle Stimulating Hormone (FSH), Luteinizing Hormone (LH), Thyroid Stimulating Hormone (TSH), renin, and testosterone were normal. Blood cultures were negative. While an abdominal computed tomography $(\mathrm{CT})$ scan demonstrated a $3.0 \times 2.7 \mathrm{~cm}$ left 

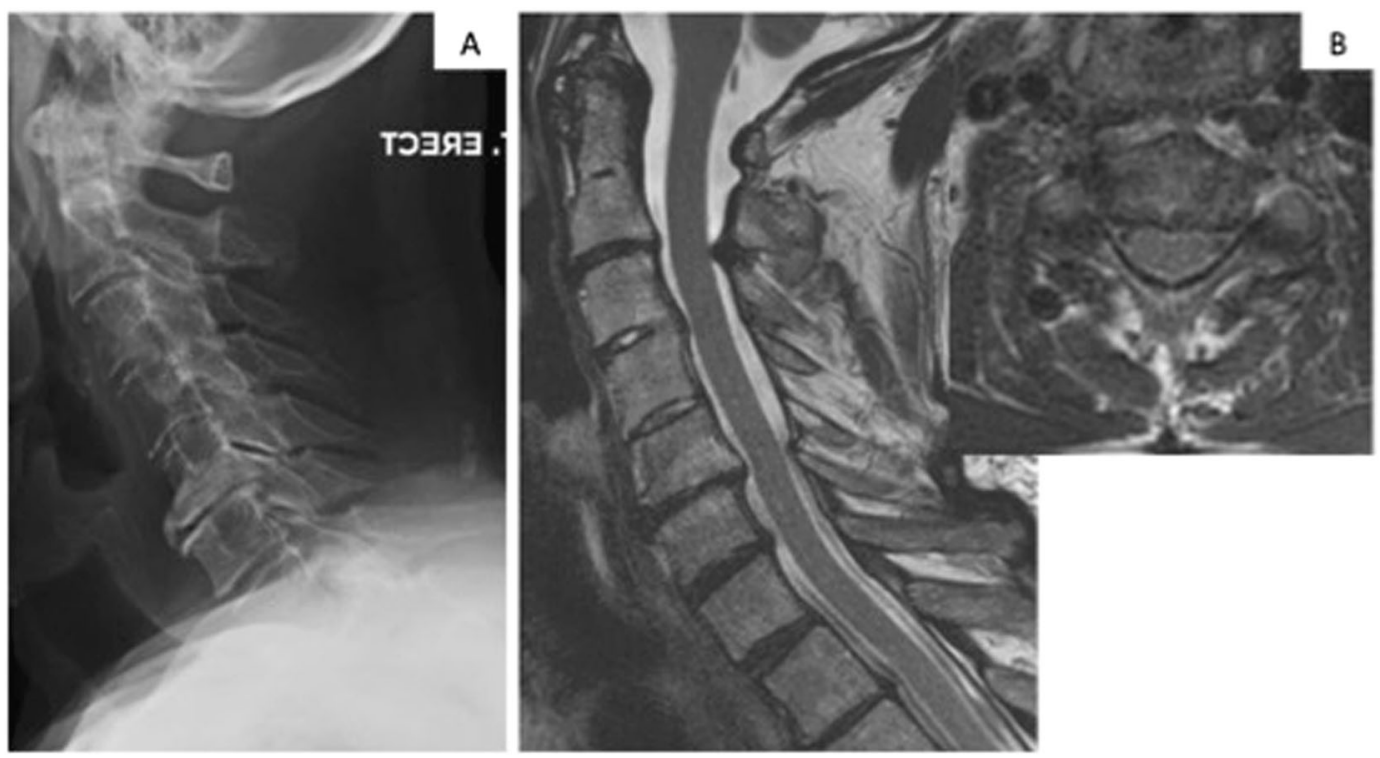

Fig. 2 Follow-up cervical spine lateral radiographs (a) and MRI (b) in 2016 demonstrate progression of multi-level degenerative disc disease with moderate/severe central and bilateral foraminal stenosis at C5-6
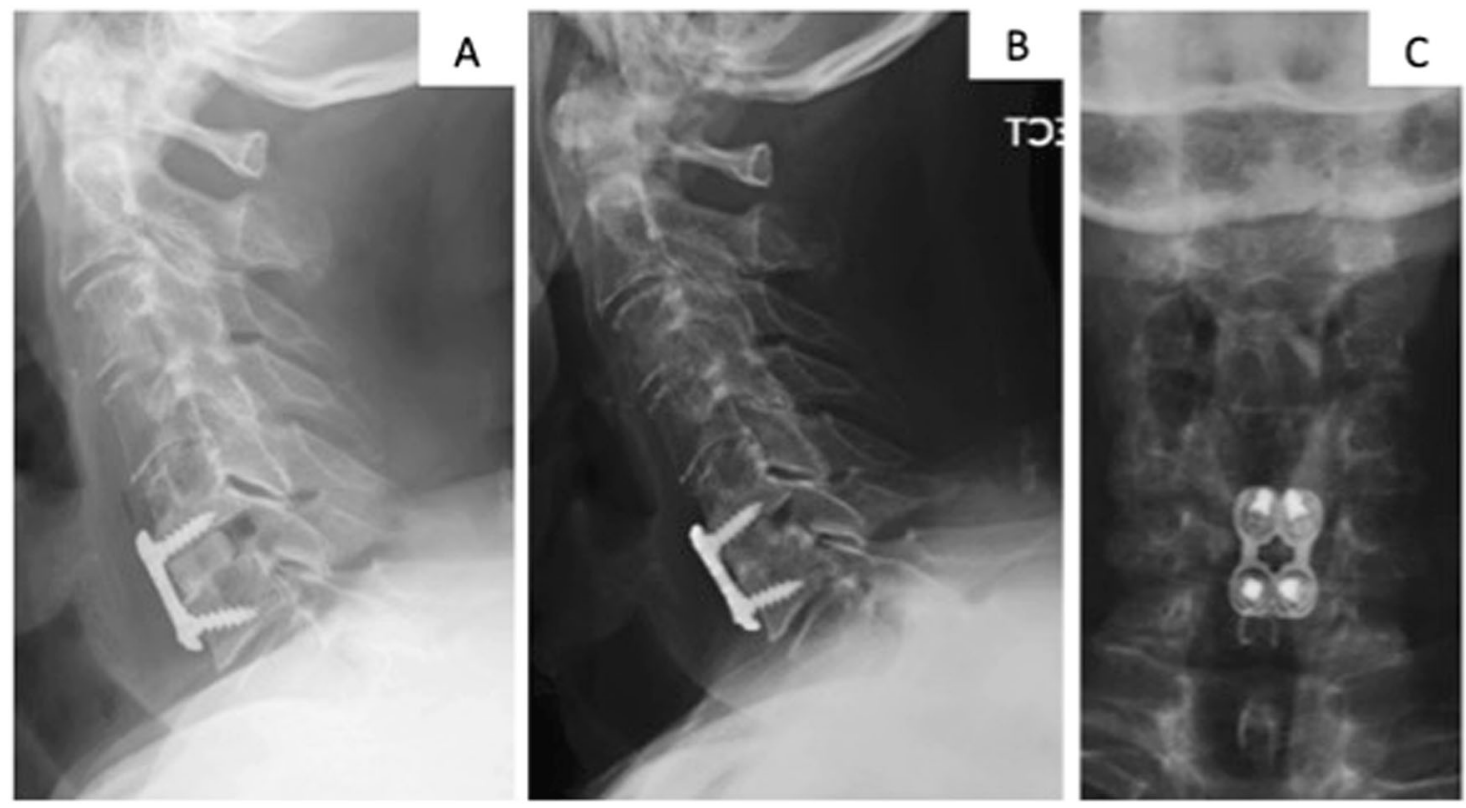

Fig. 3 Immediate post-operative (a) and 3-month follow-up (b, c) radiographs in 2016 after C5-6 anterior cervical discectomy and fusion with structural allograft and anterior plating

adrenal nodule, pheochromocytoma and Cushing's syndrome were ruled out with negative urine catecholamines, Vanillylmandelic Acid (VMA), 5-Hydroxyindoleacetic Acid (5-HIAA), cortisol aldosterone, and Adrenocorticotropic Hormone (ACTH). Subsequently, he was referred to a neurologist for further evaluation, which was notable for a positive Hoffman's on the left and $3+$ biceps, brachioradialis, and patellar reflexes bilaterally. Given his constellation of symptoms, negative endocrine work-up, and history of cervical stenosis, they attributed his symptoms to AD possibly due to his cervical stenosis. As such, he was then referred back to our spine clinic.

During the patients spine evaluation in 2016, he reported persistent and progressive symptoms suggestive of cervical myelopathy and radiculopathy, including bilateral arm pain (left $>$ right), numbness in the left thumb, and worsening balance and hand-writing. Repeat radiographs and MRI of the cervical spine demonstrated multi-level degenerative 
changes and moderate central stenosis at C5-6 that had progressed from the previous images (Fig. 2).

\section{Treatment}

Given the absence of other causes for his AD, he underwent a C5-6 anterior cervical discectomy and fusion with structural allograft and anterior plating with the goal of resolving his neurologic and autonomic symptoms. Noteworthy findings during the operation were severe bilateral foraminal stenosis and a large posterior osteophyte in the right paracentral area causing significant compression on the anterior dura. The estimated blood loss was $50 \mathrm{cc}$. There were no abnormal neuromonitoring signals throughout the entirety of the operation. Post-operatively, the patient was admitted to the Orthopedic Spine Service. The numbness in his left hand resolved and the rest of his neurologic examination was stable. Radiographs demonstrated good alignment and fixation (Fig. 3). He was discharged home on post-operative day 1 .

\section{Post surgical outcome}

During all interactions with medical teams (i.e., nurses, cardiologist, endocrinologists, spine service) post-operatively, he reported drastic improvements and resolution of his symptoms of $\mathrm{AD}$. He stated he had no diaphoresis, chills, blood pressure variations, bradycardia since surgery. At 3 months post-operatively, radiographs demonstrated good hardware alignment and progression of fusion (Fig. 3). At 6 months post-operatively, he continues to have paroxysmal atrial fibrillation, however, this is isolated and not associated with the prior constellation of symptoms. The improvement in his AD symptoms are persistent.

\section{Discussion}

$\mathrm{AD}$ is most commonly reported in physical medicine and rehabilitation literature regarding the care for patients in the chronic stage of SCIs [1, 4-6, 8-10]. In the more chronic cases, the onset of $\mathrm{AD}$ is attributable to a particular stimulation-urinary catheterization [4], bowel stimulation [11], decubitus ulcer dressing changes [12]. The majority of treatment options rely primarily on prevention of this sympathetic dysregulation. Refractory cases have been treated by sacral deafferentation to eliminate sensory stimuli [8]. There has been greater attention recently to AD in the acute phase of a SCI [6]. Although neurogenic shock is the more commonly witnessed cardiovascular phenomenon after SCI, AD can cause a paradoxical unpredictable increase in blood pressure [13]. One case report presented a patient who suffered a cerebral hemorrhage from the hypertension caused by AD [9].

There has not been any report of degenerative stenotic changes in the spine resulting in AD. The etiology of $\mathrm{AD}$ stems from injury induced dysfunction of sympathetic preganglionic neurons in the spinal cord below the level of injury. The control of these sympathetic neurons by the medullo-spinal neurons in the rostral medulla is lost due to the injury between them $[1,14]$. Thus, afferent input from visceral sensations can lead to an uninhibited sympathetic tone that leads to the constellation of symptoms characteristic of AD [15]. Given this understanding of the neural pathways, we hypothesize that our patient's cervical compression caused a disruption in the regulatory control of the sympathetic preganglionic neurons resulting in the autonomic symptoms. Although no particular stimulus was found to be causative of the episodes, any sort of visceral distension could have been responsible.

Cervical stenosis was effectively a diagnosis of exclusion in this scenario, as endocrine, cardiac, and neurological pathologies were explored and ruled out by experienced consultants in their respective fields. The amount of stenosis noted on MRI, the neurological deficits and the degree of myelopathy were quite moderate. There was also no $\mathrm{T} 2$ cord signal seen. However, the time course of the improvement in symptoms occurred subsequent to the cord decompression and an association is possible. An alternative possibility that was considered was that the anterior surgery led to transient damage to the sympathetic chain during mobilization of the longus coli, which resolved. However, damage to bilateral sympathetic chains was thought to be unlikely, and the patient showed no symptoms of Horner's syndrome.

The decision to perform an ACDF would not have been undertaken in the absence of clear neurological symptoms in the correct anatomic distributions. Recent case studies have identified Charcot spines in patients with SCI as the pathologic entity responsible for $\mathrm{AD}$, which resolves with adequate decompression and stabilization (often with fusion) [16-18]. There are some reports of spinal cord compression by tumors causing $\mathrm{AD}$ as well [19, 20]. However, from this single study it remains unclear how prominent cervical stenosis should be on the differential of patients presenting with autonomic nervous system dysfunction. The fact that the patient felt a sudden improvement in his symptoms with the near resolution of these episodes of $\mathrm{AD}$ after surgery does allow one to suspect cervical stenosis may have been the culprit, though no definitive conclusions or statements about treatment can be made from this single case.

More is being understood about possible etiologies that lead to $\mathrm{AD}$ and its underlying pathophysiology. The fact that cervical stenosis may be one of the etiologies of this 
condition warrants more investigation into the syndrome. Clinicians should be aware of the possible association between spinal stenosis and AD outside the realm of spinal trauma and SCI.

\section{Compliance with ethical standards}

Conflict of interest The authors declare that they have no competing interests.

\section{References}

1. Krassioukov A, Warburton DE, Teasell R, Eng JJ, A systematic review of the management of autonomic dysreflexia after spinal cord injury. Arch Phys Med Rehabil. 2009;90:682-95.

2. Eldahan KC, Rabchevsky AG, Autonomic dysreflexia after spinal cord injury: Systemic pathophysiology and methods of management. Auton Neurosci. 2017 [Epub ahead of print].

3. Alexander MS, Biering-Sorensen F, Bodner D, Brackett NL, Cardenas D, Charlifue S, et al. International standards to document remaining autonomic function after spinal cord injury. Spinal Cord. 2009;47:36-43.

4. Snow JC, Sideropoulos HP, Kripke BJ, Freed MM, Shah NK, Schlesinger RM, Autonomic hyperreflexia during cystoscopy in patients with high spinal cord injuries. Paraplegia. 1978;15:327-32.

5. Krassioukov A, Autonomic function following cervical spinal cord injury. Respir Physiol Neurobiol. 2009;169:157-64.

6. Krassioukov AV, Furlan JC, Fehlings MG, Autonomic dysreflexia in acute spinal cord injury: an under-recognized clinical entity. $\mathrm{J}$ Neurotrauma. 2003;20:707-16.

7. Curt a, Nitsche B, Rodic B, Assessment of autonomic dysreflexia in patients with spinal cord injury. J Neurol. 1997;62:473-7.

8. Pannek J, Göcking K, Bersch U, Sacral rhizotomy: a salvage procedure in a patient with autonomic dysreflexia. Spinal Cord. 2010;48:347-8.

9. Pan SL, Wang YH, Lin HL, Chang CW, Wu TY, Hsieh ET, Intracerebral hemorrhage secondary to autonomic dysreflexia in a young person with incomplete C8 tetraplegia: A case report. Arch Phys Med Rehabil. 2005;86:591-3.

10. Widerström-Noga E, Cruz-Almeida Y, Krassioukov A, Is there a relationship between chronic pain and autonomic dysreflexia in persons with cervical spinal cord injury?. J Neurotrauma. 2004;21:195-204.

11. Weaver LC, Marsh DR, Gris D, Brown A, Dekaban GA, Autonomic dysreflexia after spinal cord injury: central mechanisms and strategies for prevention. Prog Brain Res. 2006;152:245-63.

12. Hall PA, Young JV, Autonomic hyperreflexia in spinal cord injured patients: trigger mechanism-dressing changes of pressure sores. J Trauma. 1983;23:1074-5.

13. Furlan JC, Autonomic dysreflexia: A clinical emergency. J Trauma Acute Care Surg. 2013;75:496-500.

14. Weaver LC, Verghese P, Bruce JC, Fehlings MG, Krenz NR, Marsh DR, Autonomic dysreflexia and primary afferent sprouting after clip-compression injury of the rat spinal cord. J Neurotrauma. 2001;18:1107-19.

15. Rabchevsky AG, Segmental organization of spinal reflexes mediating autonomic dysreflexia after spinal cord injury. Prog Brain Res. 2006;152:265-74.

16. Mohit AA, Mirza S, James J, Goodkin R, Charcot arthropathy in relation to autonomic dysreflexia in spinal cord injury: case report and review of the literature. J Neurosurg Spine. 2005;2:476-80.

17. Morita M, Iwasaki M, Okuda S, Oda T, Miyauchi A, Autonomic dysreflexia associated with Charcot spine following spinal cord injury: a case report and literature review. Eur Spine J. 2010;19: S179-82.

18. Zyck S, Toshkezi G, Pizzuti J, Marawar S, Four-rod instrumentation for treatment of charcot spinal arthropathy causing autonomic dysreflexia: Case report and literature review. Cureus. 2016;8:e850.

19. Furlan J, Fehlings M, Halliday W, Krassioukov A, Autonomic dysreflexia associated with intramedullary astrocytoma of the spinal cord. Lancet Oncol. 2003;4:574-5.

20. Mathias C, Frankel H. Autonomic disturbances in spinal cord lesions. In: Bannister R, Mathias C(eds.) Autonomic failure, a textbook of clinical disorders of the autonomic nervous system. Oxford: Oxford Medical Publications; 1999. p 839-81. 